\title{
MODELO MATEMÁTICO DE LA DEMOGRAFÍA DEL GANADO DE UN PREDIO DEL SECTOR EL OCHO LETRAS
}

\author{
$\checkmark$ AlejandRo Rincón ${ }^{1}$ \\ GLORIA YANETH FLÓREZ ${ }^{1}$ \\ JOHAN MANUEL REDONDO ${ }^{2}$ \\ GERARD OLIVAR ${ }^{3}$
}

\section{RESUMEN}

En el sector El Ocho Letras la ganadería es un significativo causante de la degradación de la vegetación, humedales naturales, y el recurso hídrico. Esto conlleva a la necesidad de conocer y comprender la evolución de la cantidad de ejemplares de las diferentes edades del ganado, a lo largo del tiempo, y el efecto de las distintas variables exógenas sobre el comportamiento del sistema. A este propósito, en el presente estudio se aplica la Dinámica de Sistemas para la representación del comportamiento del número de ejemplares de ganadería del predio Laguna Negra, localizado en el sector El Ocho Letras. Se definieron como variables el número de ejemplares de ganado en las distintas edades del ciclo de vida. Los ejemplares pasan por las distintas edades, en cada una de ellas demoran un año. Para la definición de los flujos entre una y otra edad se consideraron flujos retardados. Se considera una estrategia de control para limitar el número de ejemplares mayores de un año. Se planteó un modelo matemático en tiempo discreto y se realizó simulación, obteniéndose un comportamiento oscilatorio periódico para las variables. Algunas de las principales contribuciones del trabajo están relacionadas con: análisis del efecto de variables exógenas sobre el comportamiento de las variables de nivel; planteamiento del control del número de ejemplares mayores de un año de edad basado en venta de crías hembra; desarrollo del modelo matemático a tiempo discreto, utilizando el método de cadena con flujos retardados.

PALABRAS CLAVE: Dinámica de sistemas, modelado, flujos retardados, ganado.

\section{MATHEMATICAL MODEL OF LIVESTOCK DEMOGRAPHY IN A FARM OF EL OCHO LETRAS REGION}

\section{ABSTRACT}

In El Ocho Letras region, livestock farming has significative influence on the degradation of vegetation, natural wetlands, and water resources. Thus, it is necessary to know and understand the transient behavior of the number of heads of cattle for the different age groups, and the effect of exogenous variables on the system behavior. To this end,

1 Programa de Ingeniería Ambiental. Universidad Católica de Manizales. Manizales, Colombia.

2 Departamento de matemáticas, Universidad Sergio Arboleda, Bogotá, Colombia.

3 Departamento de Ingeniería Eléctrica, Electrónica y Computación -Percepción y Control Inteligente. Manizales, Colombia

Autor de correspondencia: Rincón, A. (Alejandro). Universidad Católica de Manizales, Programa de Ingeniería Ambiental. Carrera 23 No 60-63, bloque E, Manizales, Colombia/ Tel.: (576) 8933050

Correo electrónico: arincons@ucm.edu.co
Historia del artículo:

Artículo recibido: 16-XII-2014 / Aprobado: 12-II-2015

Disponible online: 30 de enero de 2016

Discusión abierta hasta noviembre de 2016

DOI: http:/dx.doi.org/10.14508/reia.2015.12.24.101-119 (c) (i) $\rightleftharpoons$ 
System Dynamics is used in order to describe the behavior of the quantity of livestock of Laguna Negra property, located in El Ocho Letras region. Different livestock variables were defined, and each variable consists of the number of animals for a life age. The animals pass through the different ages, and they stay for one year in each. Time delays were used for the fluxes between the different ages. A control strategy was used for limiting the number of animals older than one year. A discrete time mathematical model was formulated, and simulation was performed, giving as result an oscillatory behavior for the evolution of the variables.

KEYWORDS: System Dynamics, modeling, delayed fluxes; livestock.

\section{MODELO MATEMÁTICO DA DEMOGRAFIA DO GADO DE UMA PROPRIEDADE DO SETOR EL OCHO LETRAS}

\section{RESUMO}

Na pecuária El Ocho Letras o gado é uma importante causa de degradação da vegetação, zonas húmidas naturais e recursos hídricos. Isso leva à necessidade de conhecer e compreender a evolução do número de cópias dos diferentes idades de gado, ao longo do tempo, e o efeito de diversas variáveis exógenas sobre o comportamento do sistema. A este respeito, no presente estudo se aplica a Dinâmica de Sistemas para representar o comportamento do número de cabeças de gado fazenda Laguna Negra, localizada no setor El Ocho Letras. Se definiram como variáveis o número de gado em diferentes idades do ciclo de vida. Eles passam por diferentes idades, em cada uma delas demoram um ano. É considerada Para a definição de fluxos entre uma e outra idade se consideraram fluxos retardados. Uma estratégia de controle para limitar o número de animais maiores de um ano. Um modelo matemático em tempo discreto foi levantada e simulação foi realizada para dar um comportamento oscilatório periódico para variáveis. Algumas das principais contribuições do projeto estão relacionadas a: análise do efeito de variáveis exógenas sobre o comportamento das variáveis de nível; planejamento do controle do número de animais maiores a um ano de idade com base na venda de filhotes femininas; desenvolvimento do modelo matemático de tempo discreto, usando os fluxos método cadeia retardados.

PALAVRAS-CHAVE: modelagem dinâmica de sistemas, fluxos atrasadas, gado.

\section{INTRODUCCIÓN}

Las actividades ganaderas en Colombia generan significativos impactos ambientales sobre suelo, agua, aire, energía y biodiversidad. De hecho, la actividad ganadera genera un impacto importante en ecosistemas naturales y en el recurso hídrico. El principal mecanismo de transformación de hábitats y ecosistemas en Colombia es la deforestación y su principal causa es la colonización y la expansión de la frontera agropecuaria. La ganadería bovina es la 'actividad que ocupa la mayor parte de las tierras transformadas de Colombia, afectando los ecosistemas naturales. En Colombia se ha perdido un gran porcentaje de bosques. La ganadería es la principal actividad que ha ocupado estas áreas deforestadas (Murgueitio, 1999).

En el sector el Ocho Letras hay una gran área de terreno que ha sido deforestada y ocupada principalmente por la ganadería. Los sistemas productivos de la zona El Ocho Letras comprenden sistemas agrícolas, sistemas pecuarios, actividades de extracción forestal y turismo rural. El mayor sistema productivo en la zona es la ganadería, seguida por el mono-cultivo de papa. La ganadería se da en forma extensiva que, en algunos sectores presenta un manejo semi-intensivo. Es enfocada a producción de leche y doble propósito (Fundación Pangea, 2007: pp. 29, 30, 41, 42). 
En el sector El Ocho Letras, la ganadería genera degradación significativa sobre los humedales naturales, los cuales aportan aguas a la cuenca del río Chinchiná, proporcionando recreación visual, y su espejo de agua da soporte a algunas especies animales (Fundación Pangea, 2007: pp. 29-30). La evolución de las edades del ganado, a lo largo del tiempo, incide sobre la dinámica de deforestación y la dinámica de degradación de los humedales naturales. Esto conlleva a la necesidad de conocer y comprender la evolución de la cantidad de ejemplares de las diferentes edades del ganado, a lo largo del tiempo. Sin embargo, hay ausencia de conocimiento en este sentido.

Una metodología que permite este conocimiento es la Dinámica de Sistemas, que ha mostrado ser efectiva para representar y estudiar sistemas ambientales y sistemas agropecuarios, incorporando conocimiento científico. Esta no se limita a la obtención del modelo matemático y al análisis de los resultados de simulación, sino que también permite la comprensión de: i) las causas del comportamiento de las variables características del sistema; ii) la interacción entre las variables exógenas y la interacción entre las variables características del sistema; iii) el efecto de las variables exógenas sobre las variables del sistema. La simulación con base en el modelo matemático permite establecer el comportamiento de las variables características del sistema ante diferentes escenarios de las variables exógenas (Sterman, 2000; García, 2003: p. 28). Así, la Dinámica de Sistemas es importante para comprender y analizar la dinámica de degradación de los humedales naturales en el Ocho Letras, y por tanto contribuye a plantear alternativas de manejo de la gran cantidad de ganado, de tal forma que no se realice ocupación de terreno utilizado por los humedales naturales.

En el trabajo de Schaffernicht (2006: pp. 213214), se muestra que hay casos en los cuales los individuos pasan por varias etapas, de modo que al dejar una etapa entran a la siguiente y las variables de estado son dichas etapas; entonces, se puede usar estructura de cadena. Un ejemplo de esto es cuando hay grupos de edades y los especímenes o ejemplares van pasando por cada una de las edades y al dejar un grupo de edad entran al grupo de edad subsiguiente. Por ejemplo, en la p. 215 se desarrolla un sistema de recursos humanos, usando Dinámica de Sistemas. Se considera una empresa cuyos profesionales realizan proyectos. Como variables de estado se tiene: vacantes, profesionales nuevos, y profesionales experimentados. Como variables exógenas se tiene: tasa de retiro, productividad de Expertos, productividad de Nuevos. Se tiene un comportamiento en cadena: los profesionales nuevos, después de cierto tiempo pasan a ser profesionales experimentados, y luego se retiran. Los flujos entre uno y otro nivel son flujos retardados, es decir, cada flujo es igual al anterior pero con un retardo de tiempo. La simulación resultante muestra que la evolución de «Profesionales experimentados» tiene la misma forma de «Nuevos profesionales», pero con retardo de tiempo.

En el trabajo de Dieguez y Cameroni (2014), se analizó la productividad ganadera en una explotación ganadera extensiva, ante la variación de la oferta de forraje. Para el planteamiento del modelo y la simulación se empleó el software MEGanE, en su versión actual, para plantear el modelo determinístico. Este software utiliza un modelo llamado Modelo de una Explotación Ganadera Extensiva, el cual es físico-biológico, de tipo «presa-predador», descrito por ecuaciones diferenciales en tiempo continuo. Las variables características o variables de estado son: altura del pasto (AP), peso vivo (PV). La estructura de este modelo es descrito por un diagrama causal, que indica las relaciones causa-efecto entre las variables de estado y demás variables. Se consideró como parámetro variable la cantidad máxima de forraje posible (coefClima), asociada al riesgo climático; a este se le introdujeron valores aleatorios en seis escenarios de variabilidad. Se observó que al aumentar la amplitud de la variabilidad en coefClima, como resultado se aumenta la variabilidad en la respuesta del sistema y disminuyen los valores promedio.

En el trabajo de Chajin y Jiménez (2010) se desarrollaron seis sistemas de representación del 
comportamiento de un sistema de producción bovina intensiva. Se hace uso de la metodología de Dinámica de Sistemas y Pensamiento Sistémico. El conjunto de los seis sistemas recibe el nombre de «Modelo de Simulación de Ganadería Bovina Intensiva»; y cada sistema es denominado prototipo.

Los sectores o componentes de cada prototipo son: demográfico, biofísico, productivo y económico. El conjunto de los seis sistemas se redacta en la herramienta de software SIPROBI 1.0, que a su vez se soporta en el software AGRODISI. Para cada sistema se usan ecuaciones diferenciales en tiempo continuo y la simulación se realiza a 130 meses.

El primer prototipo es llamado «Doble propósito demográfico»; permite representar la demografía de un sistema ganadero de doble propósito (producción de carne y leche); muestra los comportamientos de la población bovina. Se utilizan categorías de edad, género (hembra o macho) y estado de reproducción. Las variables de nivel o variables de estado corresponden al número de especímenes en cada categoría, número de nacimientos y número de descartes.

El segundo prototipo es llamado «Ceba intensiva demográfico y financiero»; permite representar características demográficas y financieras, de un sistema de ganadería bovina, de ceba intensiva. No se consideran hembras. La utilidad neta depende de ingresos menos egresos. Los ingresos se deben a venta de descartes y ventas de novillos cebados. Algunas de las variables de estado más importantes son: número de novillos de levante, número de novillos en ceba, número de descartes, ingresos por venta de descartes, ingresos por venta de novillos cebados, ingresos totales, egresos y utilidad neta.

El tercer prototipo es llamado «Lechería intensiva demográfico y financiero»; permite representar características demográficas y financieras de un sistema de ganadería bovina intensiva, con producción de leche. Los machos son descartados al momento de nacer. Los ingresos se deben a: venta de leche generada por las vacas en período lactante y venta de animales de descarte. Algunas de las variables de estado más importantes son: número de vacas dona- doras (las que pasan a la edad adulta y poseen una alta genética y son sometidas a fecundación), número de vacas preñadas, número de vacas preñadas lactantes, ingresos por venta de terneros, ingresos por venta de leche, ingresos por venta de descartes, ingresos por venta de embriones, ingresos totales, egresos que se deben a costos de las actividades de producción y utilidad neta.

El cuarto prototipo es llamado «componente biofísico», permite representar el rendimiento y productividad del sistema, en función de características biofísicas para un sistema de ganadería bovina intensiva. El peso vivo y el requerimiento de compra-venta de reses dependen de variables como capacidad de carga, tipo de forrajes, condiciones climáticas, y tipos de suelo. Requerimiento de compra-venta de reses es la diferencia entre capacidad de carga y peso vivo. Las variables exógenas consideradas son: i) sistema de pastoreo, ya sea pastoreo intensivo o confinamiento; ii) área, es el terreno disponible para implementar un sistema de pastoreo; iii) tipos de pasto, se consideran cuatro tipos de pasto; iv) forraje ofrecido, es la cantidad de forraje disponible en la finca; v) forraje rechazado; vi) forraje consumido; vii) energía del forraje; viii) energía total, es la energía suministrada por el forraje y por el suplemento. Algunas de las variables de estado más importantes son: compras, descartes, cantidad de pastos tipo del potrero $1 \mathrm{y}$ cantidad de pastos del potrero 2 .

El quinto prototipo es llamado «Modelo general de ceba intensiva» que consiste de la suma de los prototipos 2 y 4; permite representar características demográficas y económicas, productividad, en función de características biofísicas, de un sistema de ganadería bovina de ceba intensiva. Algunas de las variables de estado más importantes son: novillos de levante, novillos en ceba, novillos cebados, peso vivo, cantidad de animales de descarte, ingresos por venta de novillos cebados, ingresos por venta de descartes, egresos y utilidad neta. Algunas de las variable exógenas más importantes son: tipo de pasto sembrado, parea de la finca, temporadas 
de lluvia y sequía. La capacidad de carga es la diferencia entre la máxima capacidad de carga y el peso vivo y es lo que permite definir el requerimiento de compra-venta de reses.

El sexto prototipo es llamado «crecimiento del pasto y ganancia de peso»; permite representar características de ganancia de peso de un bovino, de un sistema de ganadería bovina intensiva; en función del crecimiento del pasto y la cantidad de pasto suministrado. Algunas de las variables de estado más importantes son: cantidad de pasto cortado, cantidad de pasto ensilado, peso de los animales y suplemento comprado. Algunas de las variables exógenas más importantes son: área para siembra de pasto, productividad del pasto, capacidad máxima de carga y requerimiento alimenticio de cada animal.

En el presente estudio se aplica la metodología de Dinámica de Sistemas, para describir la evolución en el número de ejemplares de ganadería en el predio Laguna Negra, localizado en el sector El Ocho Letras. Se plantea un modelo en tiempo discreto, con base en el cual se realiza simulación.

El objetivo general del trabajo es representar matemáticamente la evolución a lo largo del tiempo, de la cantidad de ganado, en el predio Laguna Negra, que está localizado en el sector el Ocho Letras. Específicamente, la cantidad de ganado comprende número de vacas, número de terneras, número de críos hembra y número de críos macho. A su vez, este objetivo comprende los siguientes objetivos específicos:

- Establecer de forma cualitativa las relaciones de influencia entre las variables exógenas y las variables endógenas, es decir, flujos, niveles y variables intermedios.

- Obtener las curvas de la evolución de las variables que se pretende estudiar, a lo largo del tiempo.

- Determinar el efecto del cambio en variables exógenas sobre la evolución temporal de las variables tipo nivel.

En el documento, la información de la sección «Compendio de datos», la sección «Definición del problema», el aparte «definición de datos para el diagrama de niveles y flujos y para la simulación» y el aparte «explicación endógena» son retomadas en partes posteriores, a saber: el aparte «Tabla de frontera de modelo», el aparte «Mapa de reservas y flujos», la sección «Formulación del modelo de simulación, tiempo discreto» y la sección «Validación del modelo». Si el lector considera innecesaria la lectura de los primeras partes, puede omitirlos.

\section{METODOLOGÍA}

El modelado por Dinámica de Sistemas, como es considerado en el presente trabajo, comprende las siguientes secciones: compendio de datos; definición del problema; formulación de la hipótesis dinámica; formulación del modelo; y validación del modelo.

La descripción de la metodología se muestra en la Tabla 1. Se planteó con base en Carvajal y Arango (2011: pp. 269-270); Carvajal, Arango, Arango y Younes (2011: pp. 61-65); Tedeschi et al. (2011); Sterman (2000: pp. 89-103); Aracil (1995: pp. 21, $36,39,41,42,58,59,65)$; Schaffernicht (2006: pp. $34-38,49,67,68,69,81,111)$.

\section{Compendio de datos}

\section{Datos generales del predio Laguna Negra}

El área pertenece a la zona amortiguadora del Parque Nacional Natural Los Nevados, municipio de Villa María (Caldas). Se encuentra a una altitud de 3 853 metros sobre el nivel del mar, con temperatura ambiental promedio de $11^{\circ} \mathrm{C}$ (Toro et al., 2012: p. 25) y una pluviosidad de $873 \mathrm{~mm}$ anuales. El clima presenta un régimen bimodal, donde los meses con más lluvia son abril y diciembre, mientras que los meses de sequía son enero y julio. El predio tiene 296 ha en total, distribuidas de la siguiente forma: 207,2 ha de ganadería y papa; 88,9 has son de conservación. Hay 30 ha de terreno en etapa de descanso, la cual se aprovecha para cultivo de papa. 
TABLA 1. DESCRIPCIÓN DE LA METODOLOGÍA

\begin{tabular}{|c|c|}
\hline Fase & Descripción \\
\hline \multirow{5}{*}{$\begin{array}{l}\text { Recolección y aná- } \\
\text { lisis de información } \\
\text { sobre el fenómeno } \\
\text { a estudiar (Sección } \\
\text { Compendio de } \\
\quad \text { datos) }\end{array}$} & $\begin{array}{l}\text { Se expresa la información sobre el área de estudio y el proceso o situación que se pretende estu- } \\
\text { diar: i) parámetros biofísicos del área de estudio, incluyendo clima, suelo, topografía, entre otros, ii) } \\
\text { datos del sistema de producción. Esta información permite entender el contexto y la situación bajo } \\
\text { estudio, y también es un insumo para que todo el estudio se realice en coherencia con la realidad. }\end{array}$ \\
\hline & $\begin{array}{l}\text { Para recolectar la información se realizó consulta bibliográfica, pero la mayor parte de la informa- } \\
\text { ción se obtuvo mediante visitas al sitio y realización de entrevistas, a las siguientes personas: i) el } \\
\text { propietario del predio, ii) un campesino de la zona, iii) dos profesores de la Universidad de Caldas. } \\
\text { Se tienen las siguientes subsecciones: }\end{array}$ \\
\hline & - $\quad$ Datos generales del predio de Laguna Negra \\
\hline & Datos demográficos \\
\hline & Venta de reses. \\
\hline \multirow{6}{*}{$\begin{array}{l}\text { Definición del } \\
\text { problema (Sección } \\
\text { Definición del Pro- } \\
\text { blema) }\end{array}$} & $\begin{array}{l}\text { Con base en la información obtenida, se definen aspectos básicos del fenómeno bajo estudio, a } \\
\text { saber: }\end{array}$ \\
\hline & _ $\quad$ El problema a afrontar, es decir, el problema ser estudiado. \\
\hline & Las variables de mayor interés para los usuarios finales de los resultados del modelo final. \\
\hline & El problema real que está a la base del problema a afrontar. \\
\hline & El propósito del modelo. \\
\hline & El horizonte de tiempo. \\
\hline \multirow{5}{*}{$\begin{array}{l}\text { Formulación de la } \\
\text { hipótesis dinámica } \\
\text { (Sección formula- } \\
\text { ción de la hipótesis } \\
\text { dinámica) }\end{array}$} & $\begin{array}{l}\text { La hipótesis dinámica es una teoría de la estructura del sistema dinámico, indica cómo surge y } \\
\text { cómo opera el sistema o la situación bajo estudio. Se plantearon las siguientes subsecciones: }\end{array}$ \\
\hline & $\begin{array}{l}\text { i) Definición de datos para el diagrama de niveles y flujos y para la simulación; se definen datos } \\
\text { y valores necesarios para desarrollar el modelo matemático y la respectiva simulación. }\end{array}$ \\
\hline & $\begin{array}{l}\text { Explicación endógena; en la cual se explica la estructura del modelo, la interacción entre } \\
\text { variables, y las causas del comportamiento de las variables; se divide en los siguientes apar- } \\
\text { tes: edades del ganado, flujos retardados, adquisición inicial de especímenes, generación } \\
\text { de nuevos críos, venta de ganado y control del número de ejemplares mayores de un año } \\
\text { de edad. }\end{array}$ \\
\hline & $\begin{array}{l}\text { Tabla de frontera del modelo: comprende la definición de variables exógenas y variables } \\
\text { endógenas; a su vez, las variables endógenas se dividen en variables tipo nivel, variables } \\
\text { tipo flujo y variables intermedias. Esta tarea es un insumo para el correcto desarrollo del } \\
\text { mapa de reservas y flujos y para el correcto planteamiento de las ecuaciones que describen } \\
\text { el sistema. }\end{array}$ \\
\hline & $\begin{array}{l}\text { iv) Mapa de reservas y flujos: son diagramas que indican principalmente las relaciones entre } \\
\text { las variables tipo nivel y las variables tipo flujo, que se forman como consecuencia de las } \\
\text { relaciones causa efecto y las realimentaciones; en él, las variables tipo nivel se representan } \\
\text { mediante rectángulos, las variables tipo flujo mediante flechas, y las variables intermedias y } \\
\text { variables exógenas no tienen ninguna forma geométrica. }\end{array}$ \\
\hline \multirow{3}{*}{$\begin{array}{l}\text { Formulación del } \\
\text { modelo de simula- } \\
\text { ción (Sección For- } \\
\text { mulación del mode- } \\
\text { lo de simulación) }\end{array}$} & $\begin{array}{l}\text { Trata de la construcción del modelo matemático. Se definen, en tiempo discreto, las ecuaciones } \\
\text { que permiten cuantificar las variables tipo nivel; a su vez, esto implica la definición de las ecuacio- } \\
\text { nes para variables intermedias y para variables tipo flujo; y definición de las condiciones iniciales. } \\
\text { Comprende las siguientes subsecciones: }\end{array}$ \\
\hline & $\begin{array}{l}\text { - Definición de flujos y ecuaciones para ganado: se definen las ecuaciones que permiten } \\
\text { cuantificar las variables correspondientes a las edades del ganado, pero también las condi- } \\
\text { ciones iniciales, las variables intermedias y los flujos. }\end{array}$ \\
\hline & $\begin{array}{l}\text { - Definición de número de críos hembra a vender: se definen las ecuaciones que permiten } \\
\text { cuantificar el número de críos hembra a vender, que es una variable intermedia que se } \\
\text { requiere para las variables de nivel. }\end{array}$ \\
\hline
\end{tabular}




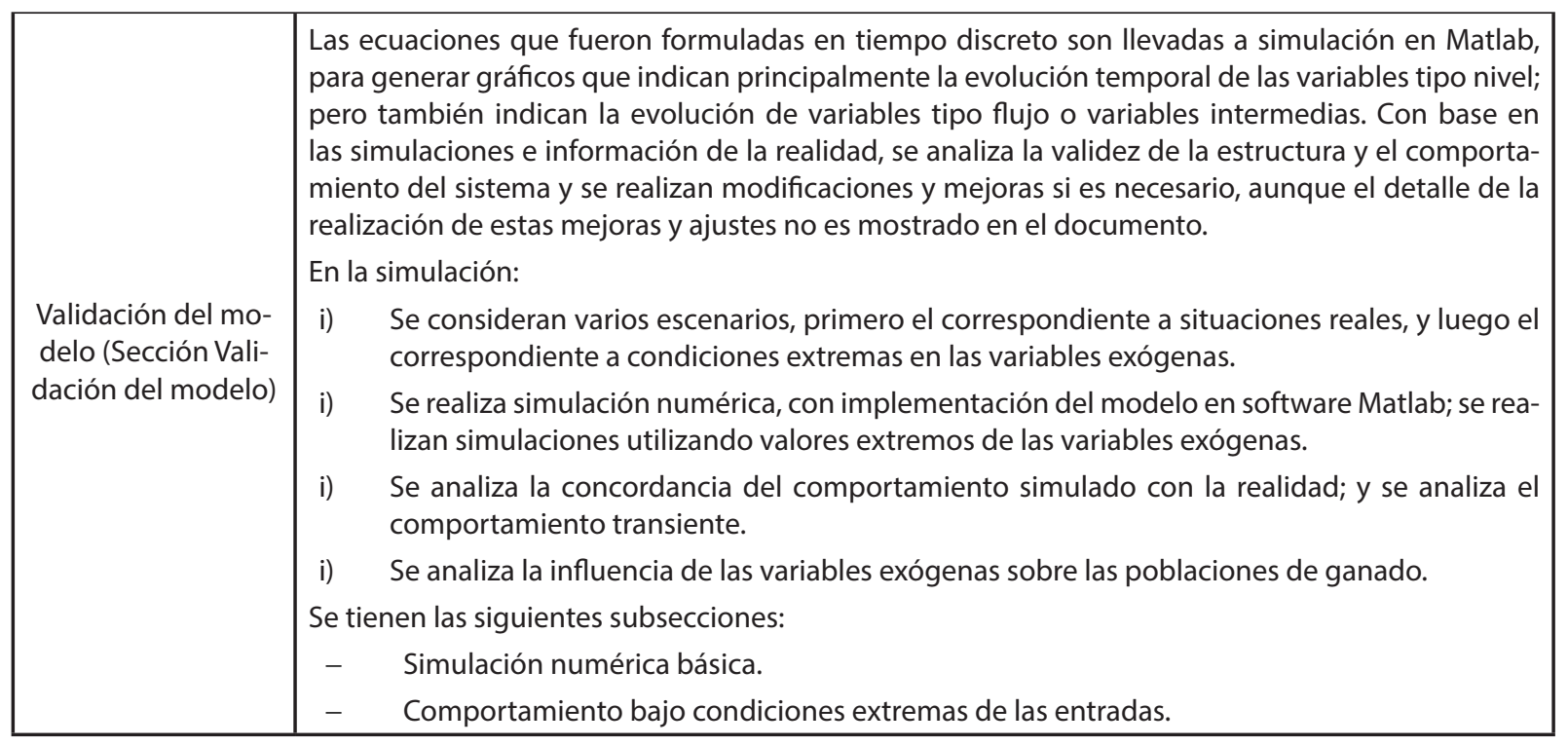

La gran altura sobre el nivel del mar implica que la disponibilidad de oxígeno es muy baja. Esto es un inconveniente significativo para la ganadería, debido a que el animal debe hacer un alto nivel de esfuerzo para lograr el oxígeno requerido para las células; el rendimiento de productividad de leche y de carne es menor, y todos los procesos son más lentos. La calidad nutricional del pasto es baja en la zona de Laguna Negra debido a esto. Además, la baja pluviosidad del lugar hace que la producción de pasto sea baja. Se realiza pastoreo rotacional, que consiste en alternar el uso de potreros. Este se realiza cada 20 días.

\section{Datos demográficos}

El ganado del predio es todo de tipo Normando, el cual es de doble propósito: para producir leche y carne de alta calidad. Es una raza que se caracteriza por: i) presenta capacidad de recorrer terrenos extensos y escarpados en búsqueda de alimento; y ii) presenta alta resistencia a las enfermedades, de modo que la mortandad es despreciable.

En el predio hay 200 vacas aproximadamente, lo cual implica una razón de una res por hectárea, aproximadamente. La vida productiva del ganado comienza a los 3 años de edad, aproximadamente. La frecuencia de los partos que sufre una res es un ternero por año, aproximadamente. Normalmente hay un lapso de tiempo de 365 días cada parto. Para el porcentaje de hembras en los nacimientos, se puede utilizar un valor de $50 \%$. El período de gestación de una res es de 9 meses. La lactancia toma 300 días aproximadamente, se desteta el ternero a los 7 meses. Luego, 60 días antes del nuevo parto la vaca es secada, es decir, se detiene el ordeño. Una cría hembra demora 24 meses para volverse una vaca adulta. A los 24 meses se le dispone un toro para que empiece a generar terneras $y$, por consiguiente, produzca leche. No es necesario comprar terneras, pues las vacas generan sus terneras, para lo cual es necesario hacer preñamiento. Generalmente, cuando una res alcanza entre 5 y 7 partos se vende para carne. De este modo, se vende la res a los 8 años de edad, aproximadamente.

\section{Venta de reses}

Hay un límite para la cantidad de ejemplares de ganado que pueden ocupar un terreno, que está dado por el área disponible y corresponde a 1 res/ ha, aproximadamente. Este límite comprende ejemplares macho y hembra mayores a un año de edad. Cuando el número de ejemplares mayores de un año de edad alcanza dicho límite, con el fin de no sobrepasar el límite no se introducen más reses y adicionalmente se debe vender reses, según las siguientes 
opciones: i) venta de terneros y terneras de 1 semana de edad; y ii) venta de vacas de descarte. Las vacas de descarte son los animales que por su edad presentan una bajo nivel de producción de leche, por tanto son vendidas para carne. Para la venta de ejemplares macho hay dos opciones: i) venderlos a la edad de una semana; o ii) realizar destete a los 7-8meses, cebar y venderlos a los 3 años de edad.

\section{Definición del problema}

El problema a ser estudiado y representado es la actividad ganadera, incluyendo generación de ganado para venta. Las variables que se pretende estudiar son: cantidad de ganado en general, cantidad de vacas productivas, cantidad de ejemplares macho y cantidad de terneras. La actividad ganadera genera una ganancia económica significativa, lo cual es la motivación a utilizar el suelo para ganadería. Esto implica la utilización de areas inicialmente ocupadas por especies vegetales, incluyendo áreas de humedales naturales.

El propósito del modelo es describir la evolución a lo largo del tiempo, de la cantidad de ganado, en el predio Laguna Negra, que está localizado en el sector el Ocho Letras; incluyendo número de vacas, número de terneras, número de críos hembra y número de críos macho. Se tiene en cuenta el efecto del cambio en variables exógenas sobre la evolución, a lo largo del tiempo, de las variable tipo nivel.

Para el horizonte de tiempo se tiene en cuenta los siguientes factores o datos:

- En Bozic (2009: p. 50) se muestra en una gráfica de la evolución del número de vacas lecheras, se toma una lapso de 1975 a 2005, es decir 30 años; pero en lapsos de 20 años se notan cambios apreciables.

- En Bozic (2009: p. 16) se muestra en una gráfica de la evolución del número de vacas lecheras en USA, se toma un lapso de 1950 a 2005, es decir 55 años, pero en lapsos de 20 años se notan cambios apreciables.

Por tanto, se toma un horizonte de tiempo de 25 años.

\section{Formulación de la hipótesis dinámica}

Esta sección comprende: i) definición de datos necesarios para el diagrama de niveles y flujos y para la simulación; ii) explicación endógena; iii) tabla de frontera del modelo: variables endógenas, variables exógenas; iv) mapa de reservas y flujos; v) discusión y conclusiones.

Definición de datos para el diagrama de niveles y flujos y para la simulación

Se toman los siguientes datos, para facilitar la representación del diagrama de niveles y flujos y para la simulación: i) edad a la que los ejemplares hembra tienen su primera cría: a los 3 años de edad; ii) porcentaje hembras en los nacimientos: $50 \%$; iii) número límite de ejemplares mayores a un año de edad: 200; iv) edad de venta de las vacas para carne: a los 8 años de edad, son vendidas sin tener su sexta cría; v) edad de venta de ejemplares macho: a una semana de edad; vi) edad de venta de ejemplares hembra: a una semana de edad; vii) tiempo de simulación: 60 años.

\section{Explicación endógena}

Las variables de ganado más importantes son: i) terneras, es decir ejemplares hembra entre 0 y 3 años de edad; ii) vacas productivas, las cuales generan leche, presentan una edad entre 3 y 8 años; iii) número de críos macho, iv) número de críos hembra.

Edades del ganado. Se establecieron diferentes edades de ganado en lugar de tener una sola, debido a que cada una de estas genera distintos efectos. Los ejemplares hembra fueron divididos en varias edades, que van desde terneras tipo 1 a vacas tipo 5. En las terneras se consideran tres edades, y en las vacas se consideran cinco edades. Cada una de estas edades tiene una duración aproximada de un año. Es decir, un espécimen, al entrar a ser ternera o vaca, se demora un lapso de un año en la edad a la cual entra, al cabo de la cual pasa a la siguiente. Los nuevos ejemplares adquiridos entran a la etapa de terneras tipo 1, etapa en la cual se demoran un año. Luego pasan a terneras 2 , luego a terneras tipo 
3 , luego a vacas tipo 1 , luego a vacas tipo 2 , vacas tipo 3 , vacas tipo 4 , vacas tipo 5 , luego son vendidos para carne. En cada una de estas etapas se demoran un año. La diferencia entre vacas y terneras es que las vacas tienen críos y producen leche. Los ejemplares vacas 1 hasta vacas 5 son los que producen leche, la cual genera la principal ganancia económica. La variable «Número de vacas» $\left(N_{v c}\right)$ se refiere al número de vacas que producen leche y se define como la suma del número de vacas desde número de vacas tipo $1\left(N_{v c 1}\right)$ hasta número de vacas $5\left(N_{v c 5}\right)$. De este modo, no tiene en cuenta el número de terneras ni de críos. La variable «Número de ejemplares 1» $\left(N_{e j e 1}\right)$, se refiere a número de ejemplares mayores a un año de edad, y se define como la suma de «Número de Terneras 2» $\left(N_{t n r 2}\right)$, «Número de Terneras 3» $\left(N_{t n r 3}\right)$, «Número de Vacas 1 ' $\left(N_{v c 1}\right), \ldots$, Número de Vacas $5 »\left(N_{v c 5}\right)$. Es decir, no tiene en cuenta «Número de Terneras tipo 1» $\left(N_{t n r 1}\right)$, pues esta última corresponde al rango 0 a 1 años de edad.

Flujos retardados. Los flujos entre las distintas edades son flujos retardados: el flujo $f_{o b}$ es igual a $f_{o}$ retardado un año; el flujo $f_{o c}$ es igual a $f_{o b}$ retardado un año; el flujo $f_{1}$ es igual a $f_{o c}$ retardado un año; el flujo $f_{1 b}$ es igual a $f_{o c}$ retardado un año; el flujo $f_{1 c}$ es igual a $f_{1 b}$ retardado un año; el flujo $f_{1 d}$ es igual a $f_{1 c}$ retardado un año; el flujo $f_{1 e}$ es igual a $f_{1 d}$ retardado un año. Un ejemplo o fenómeno similar de flujos retardados se puede encontrar en el texto de Schaffernicht (2006: pp. 217 y 218).

Adquisición inicial de especímenes. En un principio, en tiempo inicial $\left(t_{o}\right)$, aparecen terneras por compra. Solo se compran Terneras tipo 1 y solo se realiza compra en el tiempo inicial. En momentos posteriores al tiempo inicial, los ejemplares de Terneras tipo 1 no aparecen como consecuencia de la compra, sino que aparecen a partir de vacas preñadas pertenecientes a la misma finca. A su vez, estas vacas han aparecido como consecuencia de las terneras compradas en un inicio. La compra inicial de terneras es lo que detona o inicia la evolución temporal de las variables.
Generación de nuevos críos. La generación de críos ocurre aproximadamente cada año. De dichos críos hay un porcentaje de hembras y un porcentaje de machos. Los críos macho pasan a ser vendidos. De los críos hembra, parte son vendidas con el fin de limitar el número de ejemplares mayores de un año de edad. Los críos hembra que no son vendidas pasan a ser Terneras tipo 1. Cuando entra un nuevo ejemplar a ser Vaca tipo 1, este ejemplar genera un nuevo crío, que puede ser macho o hembra. Así mismo, al entrar un nuevo ejemplar a ser «Vaca tipo 2», «Vaca tipo 3», «Vaca tipo 4» o «Vaca tipo 5», se genera un nuevo crío que puede ser macho o hembra. Si el nuevo crío es macho, entonces se vende a la edad de una semana. Pero si el crio es hembra, puede entrar a la etapa «Ternera tipo 1» o puede ser vendida si se requiere para limitar el número de ejemplares mayores de un año de edad.

Venta de ganado. Los ejemplares hembra, después de ser Vaca tipo 5 completan los ocho años de edad y pasan a ser vendidos para carne. Los ejemplares macho son vendidos cuando alcanzan una semana de edad. La cantidad de ejemplares mayores de un año de edad no puede sobrepasar un valor límite que está dado por la cantidad de área disponible. A este fin contribuye la venta de vacas de 8 años de edad y la venta de críos macho de una semana de edad; pero no es suficiente, por tanto se realiza venta de una parte de los críos hembra, que tienen una semana de edad. El número de críos hembra vendidos se decide de tal forma que el número de ejemplares mayores a un año de edad no sobrepase el valor límite.

\section{Control del número de ejemplares mayores}

de un año. La definición de dicho control es una tarea compleja que implicó analizar varias opciones y como resultado se seleccionó la siguiente opción: realizar venta de críos hembra. Esta opción permite: i) mantener un alto número de vacas tipo 1 a tipo 5 , por tanto manteniendo una alta producción de leche; ii) obtener números enteros en el desarrollo del algoritmo de simulación en Matlab. Además, presenta la siguiente característica: la venta de crías 
hembra debe hacerse de manera anticipada, es decir, definiendo el número de críos hembra que se debe vender en un momento $k$, de tal forma que en un momento $k+1$ no haya exceso en el número de ejemplares mayores de un año de edad.

\section{Tablas de frontera de modelo}

Comprende variables endógenas y variables exógenas. A su vez, las variables endógenas se clasifican en variables tipo flujo, variables tipo nivel y variables intermedias.

\section{Variables tipo nivel:}

- Número de Terneras tipo 1: número de especímenes hembra de ganado vacuno de entre cero y un año de edad, presentes en la finca $\left(N_{t n r 1}\right)$.

- Número de Terneras tipo 2: número de especímenes hembra de ganado vacuno de entre uno y dos años de edad, presentes en la finca $\left(N_{t n r 2}\right)$.

- Número de Terneras tipo 3: número de especímenes hembra de ganado vacuno de entre dos y tres años, presentes en la finca $\left(N_{t n r 3}\right)$.

- Número de vacas tipo 1: número de vacas presentes en la finca que solo han tenido la primera cría y tienen una edad entre tres y cuatro años $\left(N_{v c 1}\right)$.

- Número de vacas tipo 2: número de vacas presentes en la finca que han tenido la segunda cría y tienen una edad entre cuatro y cinco años $\left(N_{v c 2}\right)$.

- Número de vacas tipo 3: número de vacas presentes en la finca que han tenido la tercera cría y tienen una edad entre cinco y seis años $\left(N_{v c 3}\right)$.

- Número de vacas tipo 4: número de vacas presentes en la finca que han tenido la cuarta cría y tienen una edad entre seis y siete años $\left(N_{v c 4}\right)$.

- Número de vacas tipo 5: número de vacas presentes en la finca que han tenido la quinta cría y tienen una edad entre siete y ocho años $\left(N_{v c 5}\right)$.

\section{Variables tipo Flujo:}

- $f_{o}$ es la velocidad de entrada de terneras tipo 1 en la finca;

- $f_{a}$ es la velocidad de entrada de terneras tipo 1 en la finca, adquiridas por compra;
- $f_{o b}$ es la velocidad de aparición de terneras tipo 2 en la finca;

- $f_{o c}$ es la velocidad de aparición de terneras tipo 3 en la finca;

- $f_{1}$ es la velocidad de aparición de vacas tipo 1 en la finca;

- $f_{1 b}$ es la velocidad de aparición de vacas tipo 2 en la finca;

- $f_{1 c}$ es la velocidad de aparición de vacas tipo 3 en la finca;

- $f_{1 d}$ es la velocidad de aparición de vacas tipo 4 en la finca;

- $f_{1 e}$ es la velocidad de aparición de vacas tipo 5 en la finca;

- $f_{1 f}$ es la velocidad de desaparición de vacas tipo 5 de la finca, debido a su venta para carne.

\section{Variables intermedias:}

- Número de críos macho: es el número de ejemplares recién nacidos macho.

- Número de críos hembra.

- Número de vacas productivas $\left(N_{v c}\right)$ : es el número de vacas activas, lo que comprende «Número de vacas tipo 1 » hasta «Número de vacas tipo 5».

- Número de ejemplares tipo $1\left(N_{\text {eje1 } 1}\right)$ : número de ejemplares mayores a un año de edad

- Número de críos hembra para venta $\left(N_{c h b}\right.$. vent): es el número de críos hembra a ser vendidos.

- Número de prueba futuro $\left(N_{p b f t}\right)$ : es el número de ejemplares mayores de un año, para un momento futuro $k+1$, pero calculado en el momento $k$, bajo la suposición de que no hay venta de críos hembra.

\section{Variables exógenas:}

- Número de terneras compradas en tiempo inicial to: es la compra de terneras, tipo 1 , en el tiempo inicial $\left(N_{\text {tnrto }}\right)$. Está relacionado con la superficie de ganadería en tiempo inicial $t=t_{0}$.

- Porcentaje de nacimientos de hembra en los partos de las vacas; 
- Número límite de especímenes mayores de un año de edad.

\section{Mapa de reservas y flujos}

En la Figura 1 se muestra el mapa de reservas y flujos. En este mapa se ubican las variables tipo nivel en forma de rectángulos; las variables tipo flujo mediante flechas; y para las variables intermedias no se utiliza ninguna forma geométrica (v. Carvajal, 2011: p., 270; Sterman, 2000). La venta de críos hembra implica un lazo de realimentación de tipo balance, de tal forma que se evita un crecimiento del número de ejemplares hacia el infinito. Ver discusión en Tedeschi, Nicholsony Rich (2011: p. 105).

\section{Formulación del modelo de simulación,}

\section{tiempo discreto}

El número de terneras, vacas productivas y machos se comporta a manera de pulsos, por tanto es más sencillo realizar un modelado a tiempo discreto que en tiempo continuo. La presencia de los flujos retardados hace complejo el modelado en tiempo continuo (Aracil, 1999: p. 12). En contraste, en tiempo discreto es altamente simple obtener el valor retardado de cualquier variable y el método es utilizar índices para designar y guardar estos valores. Por tanto, se realizó modelado en tiempo discreto, usando Matlab.

\section{Definición de flujos y ecuaciones para ganado}

Ya que se está considerando tiempo discreto, se utiliza un índice de conteo, denominado k, y el tiempo inicial se define como $k=1$. Se debe tener en cuenta que: i) inicialmente se compran terneras tipo 1 por parte del propietario, se asume un valor de 50 terneras tipo 1; ii) inicialmente no se adquieren vacas para la obtención de leche, pues en lugar de esto se adquieren terneras tipo 1 que posteriormente crecen para volverse vacas y producir leche. Por tanto, el número de Terneras 2, Terneras 3, Vacas 1,... Vacas 5 es cero en tiempo inicial. Además, en tiempo inicial $k=1$, solo el flujo de entrada de Terneras 1 es diferente de cero, los demás flujos son cero. El flujo de compra de terneras es un pulso que se define como:

$$
\left.f_{\alpha}\right|_{k=1}=50,\left.f_{\alpha}\right|_{k>1}=0
$$

Los flujos para terneras y vacas son flujos retardados, como se había mencionado en la parte de «Explicación endógena», «flujos retardados». Para esto nos basamos en parte en el texto de Schaffernicht (2006: pp. 217- 218).

Figura 1. Diagrama de niveles y flujos.

Fuente: elaboración propia de los autores, utilizando el software Vensim.

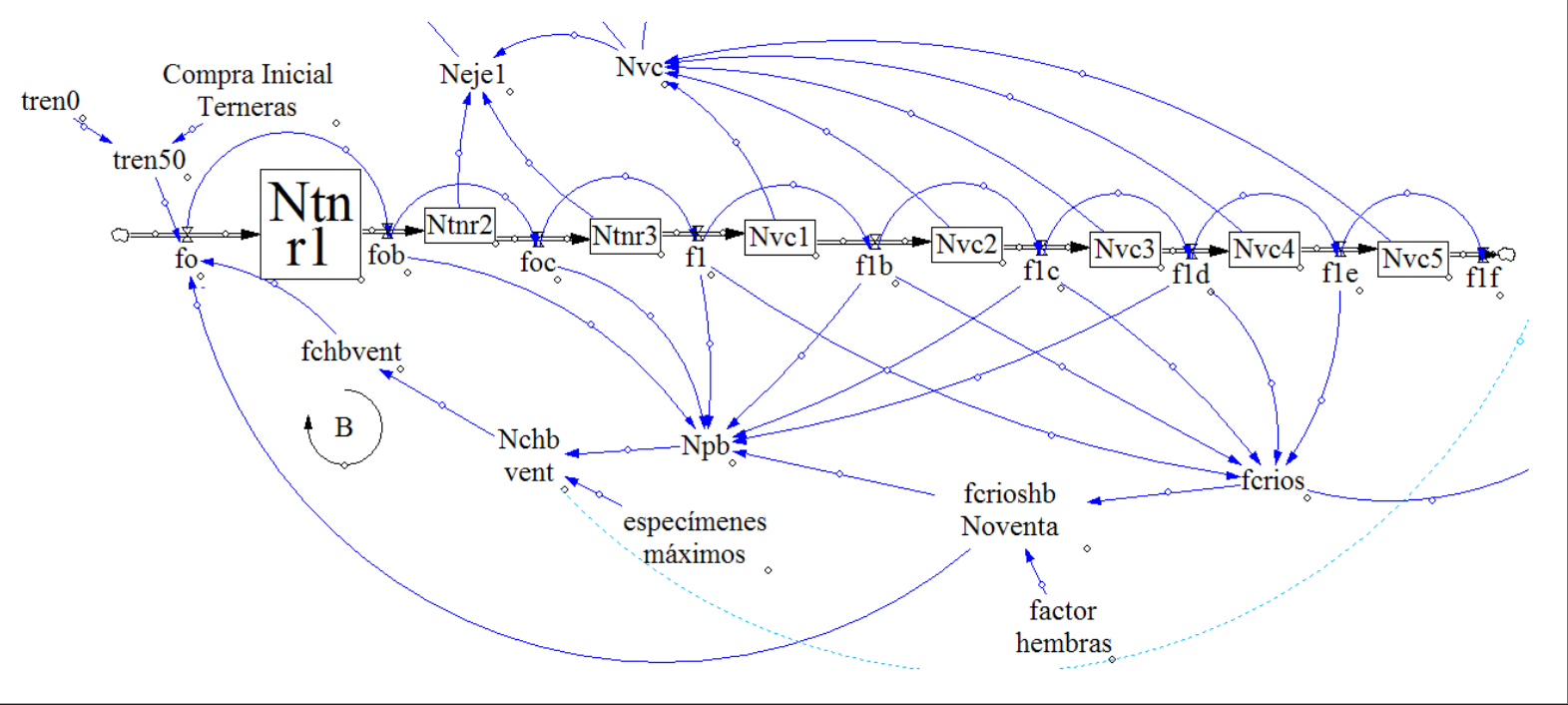


Para calcular el flujo de entrada de terneras tipo 1, representado por $f_{o^{\prime}}$ se necesita: i) número de críos hembra que son vendidos con el fin de evitar que el número de ejemplares mayores de un año sobrepase el valor límite: $\left.N_{c h b v e n t}\right|_{k}$;ii) el porcentaje de nacimientos hembra en los partos de las vacas: $N_{\text {ncmhb }}$; iii) número de críos, incluyendo machos y hembra: $\left(\left.f_{1}\right|_{k}+\left.f_{1 b}\right|_{k}+\left.f_{1 c}\right|_{k}+\left.f_{1 d}\right|_{k}+\left.f_{1 e}\right|_{k}\right) ;$ iv) entrada de Terneras tipo 1 por compra: $f_{a}$ El flujo $f_{a}$ sólo toma un valor distinto de cero en el tiempo inicial. El flujo $f_{0}$ cumple la siguiente ecuación:

$\left.f_{0}\right|_{k}=\left.f_{\alpha}\right|_{k}+N_{n c m h b}\left(\left.f_{1}\right|_{k}+\left.f_{1 b}\right|_{k}+\left.f_{1 c}\right|_{k}+\left.f_{1 d}\right|_{k}+f_{1 e}||_{k}\right)-\left.N_{c h b v e n t}\right|_{k}$

El número de ejemplares de cada una de las distintas edades, es igual al flujo de entrada a la edad correspondiente:

$$
\begin{aligned}
& \left.N_{t n r 1}\right|_{k}=\left.f_{O}\right|_{k},\left.N_{t n r 1}\right|_{k=1}=\left.\left.f_{\alpha}\right|_{k} \quad N_{t n r 2}\right|_{k}=\left.f_{o b}\right|_{k},\left.N_{t n r 2}\right|_{k=1}=0 ; \\
& \left.N_{t n r 3}\right|_{k}=\left.f_{o c}\right|_{k} \text {, } \\
& \left.N_{t n r 3}\right|_{k=1}=\left.0 \quad N_{v c 1}\right|_{k}=\left.f_{1}\right|_{k},\left.N_{v c 1}\right|_{k=1}=\left.0 \quad N_{v c 2}\right|_{k}=\left.f_{1 b}\right|_{k}, \\
& \left.N_{v c 2}\right|_{k=1}=0 \\
& \left.N_{v c 3}\right|_{k}=\left.f_{1 c}\right|_{k},\left.N_{v c 3}\right|_{k=1}=\left.0 \quad N_{v c 4}\right|_{k}=\left.f_{1 d}\right|_{k},\left.N_{v c 4}\right|_{k=1}=0 \\
& \left.N_{v c 5}\right|_{k}=\left.f_{1 e}\right|_{k},\left.N_{v c 5}\right|_{k=1}=0
\end{aligned}
$$

\section{Definición del número de críos hembra a vender}

Se venden críos hembra de tal forma que el número de ejemplares mayores de un año de edad no sobrepase el valor límite.

En cualquier momento dado, los ejemplares mayores a un año de edad incluyen desde terneras tipo 2, que son terneras de entre uno y dos años de edad, hasta vacas tipo 5. Resulta que las terneras tipo 2, antes de ser tipo 2, un año atrás han pasado por la etapa de tipo 1 y también han pasado por la etapa de críos hembra. Por tanto, si en un momento dado $k$, se vende parte de los críos hembra, se logra reducir el valor que en un momento futuro $k+1$ tomaría el número de terneras tipo $2 \mathrm{y}$, por tanto, el número de ejemplares mayores de un año de edad.

Se tomó esta opción de vender parte de los críos hembra, pues así se logra reducir de forma directa el exceso de críos hembra y se mantiene un alto número de vacas productivas $N_{v c^{\prime}}$ evitando valores excesivos del número de ejemplares de terneras tipo 2 y terneras tipo 3. Además, es una opción coherente con la realidad.

Se considera un índice de prueba, $N_{p b f t}$ el cual indica, en un momento $k$, el número de ejemplares mayores de un año de edad, para un momento futuro $k+1$, bajo la condición de no venta de críos hembra.

$$
\begin{aligned}
& \left.N_{p b f t}\right|_{k}=\left.N_{t n r 2}\right|_{k+1}+\left.N_{t n r 3}\right|_{k+1}+\left.N_{v c 1}\right|_{k+1}+\left.N_{v c 2}\right|_{k+1}+\left.N_{v c 3}\right|_{k+1}+ \\
& \left.\quad N_{v c 4}\right|_{k+1}+\left.N_{v c 5}\right|_{k+1} \\
& =\left.f_{o b}\right|_{k+1}+\left.f_{o c}\right|_{k+1}+\left.f_{1}\right|_{k+1}+\left.f_{1 b}\right|_{k+1}+\left.f_{1 c}\right|_{k+1}+\left.f_{1 d}\right|_{k+1}+\left.f_{1 e}\right|_{k+1} \\
& =\left.f_{o}\right|_{k}+\left.f_{o b}\right|_{k}+\left.f_{o c}\right|_{k}+\left.f_{1}\right|_{k}+\left.f_{1 b}\right|_{k}+\left.f_{1 c}\right|_{k}+\left.f_{1 d}\right|_{k}
\end{aligned}
$$

Anteriormente se definió el flujo de entrada de Terneras $1,\left.f_{o}\right|_{k^{\prime}}$ lo cual incluye el término $\left.N_{\text {chbvent }}\right|_{k^{\prime}}$ el cual corresponde a la venta de críos hembra. Cuando no hay venta de críos hembra, es decir, $\left.N_{c h b v e n t}\right|_{k}=0$, la entrada de Terneras tipo 1 es:

$$
\left.f_{o}\right|_{k}=\left.f_{\alpha}\right|_{k}+N_{n c m h b}\left(\left.f_{1}\right|_{k}+\left.f_{1 b}\right|_{k}+\left.f_{1 c}\right|_{k}+\left.f_{1 d}\right|_{k}+\left.f_{1 e}\right|_{k}\right)
$$

Reemplazando esta expresión de $\left.f_{o}\right|_{k^{\prime}}$ en la expresión de $\left.N_{p b f t}\right|_{k^{\prime}}$ se obtiene:

$$
\begin{aligned}
N_{p b f t}=\left.f_{\alpha}\right|_{k} & +N_{n c m h b}\left(\left.f_{1}\right|_{k}+\left.f_{1 b}\right|_{k}+\left.f_{1 c}\right|_{k}+\left.f_{1 d}\right|_{k}+\left.f_{1 e}\right|_{k}\right)+\left.f_{o b}\right|_{k} \\
& +\left.f_{o c}\right|_{k}+\left.f_{1}\right|_{k}+\left.f_{1 b}\right|_{k}+\left.f_{1 c}\right|_{k}+\left.f_{1 d}\right|_{k}
\end{aligned}
$$

La anterior ecuación permite el cálculo del valor de $N_{p b f t}$ en la simulación. Se define el excedente, es decir, el sobrante de ejemplares mayores a un 
año de edad, el cual ocurriría si no hay venta de críos hembra:

$$
N_{p b f t}-N_{\text {lim }}
$$

Este excedente sólo se calcula para cuando $N_{p b}$ ft es mayor al número límite $N_{\text {lim }}$. Se tienen dos casos: i) $N_{p b f t}$ es mayor al número límite $N_{\text {lim }}$, en este caso, se deben vender críos hembra, de modo que el número de crías hembra a vender es mayor a cero, $\left.N_{c h b v e n t}\right|_{k}>0$; ii) $N_{p b f t}$ es menor o igual al número límite $N_{\text {lim }}$ en este caso, no se debe vender críos hembra, de modo que el número de crías hembra a vender es cero, $\left.N_{\text {chbvent }}\right|_{k}=0$.

El número de críos hembra a vender es igual al excedente:

$$
\left.N_{c h b v e n t}\right|_{k}=N_{p b f t}-N_{\text {lim }}, \text { Si } N_{p b f t}>N_{\text {lim }}
$$

De esta forma, la lógica del algoritmo es:

$$
\begin{aligned}
& N_{p b f t}=f_{\left.\alpha\right|_{k}}+N_{n c m h b}\left(\left.f_{1}\right|_{k}+\left.f_{1 b}\right|_{k}+\left.f_{1 c}\right|_{k}+\left.f_{1 d}\right|_{k}+\left.f_{1 e}\right|_{k}\right)+\left.f_{o b}\right|_{k}+ \\
& \left.f_{o c}\right|_{k}+\left.f_{1}\right|_{k}+\left.f_{1 b}\right|_{k}+\left.f_{1 c}\right|_{k}+\left.f_{1 d}\right|_{k}
\end{aligned}
$$

Si $N_{\text {pbft }} \leq N_{\text {lim }}$

$$
\text { entonces }\left.N_{\text {chbvent }}\right|_{k}=0
$$

sino

$$
\text { entonces }\left.N_{\text {chbvent }}\right|_{k}=N_{\text {pbft }}-N_{\text {lim }}
$$

fin

$\left.f_{o}\right|_{k}=\left.f_{\alpha}\right|_{k}+N_{n c m h b}\left(\left.f_{1}\right|_{k}+\left.f_{1 b}\right|_{k}+\left.f_{1 c}\right|_{k}+\left.f_{1 d}\right|_{k}+\left.f_{1 e}\right|_{k}\right)-\left.N_{c h b v e n t}\right|_{k}$

\section{Validación del modelo}

En esta sección, las ecuaciones formuladas en tiempo discreto son llevadas a simulación en el software Matlab. Esta sección comprende: i) simulación numérica básica; ii) comportamiento bajo condiciones extremas de las entradas; iii) discusión y conclusiones.

\section{Simulación numérica básica}

Se utilizó el software Matlab. En la Figura 2 se muestra la manera como se corresponde el índice $\mathrm{k}$ con el tiempo, para el caso que se utiliza modelado en tiempo discreto.

Figura 2. Relación entre el índice $k$ y el tiempo, para el caso que se utiliza modelado en tiempo discreto.

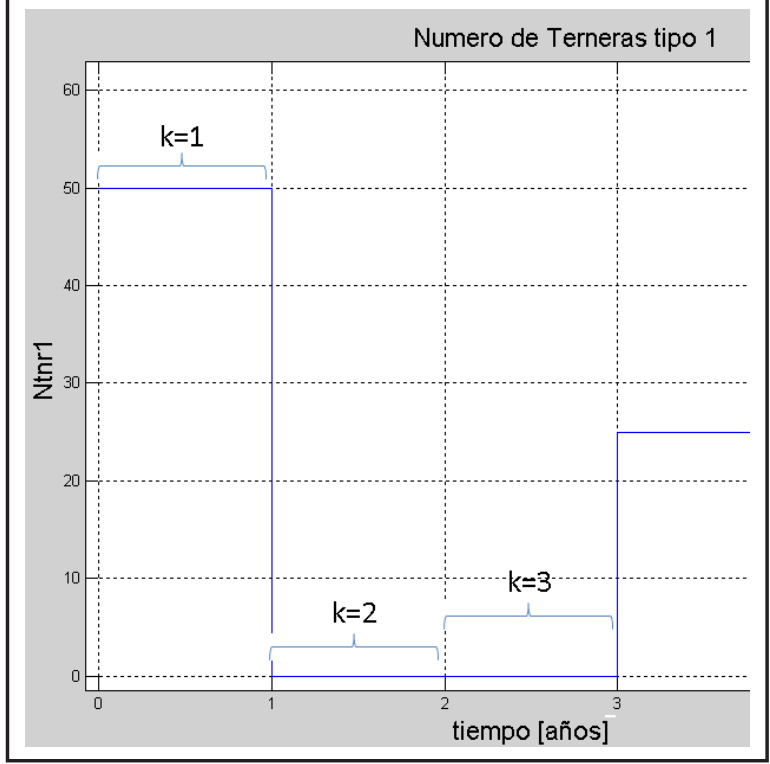

En la Figura 3 se presenta la evolución del número de Terneras tipo $1\left(N_{t n r 1}\right)$ y número de Terneras tipo $2\left(N_{t n r 2}\right)$. Una primera característica que se puede notar es que el valor de $N_{t n r 1}$ comienza en 50, esto se debe a que este es el número de especímenes comprados en el tiempo inicial. Una segunda característica es que $N_{t n r 1}$ se hace cero y luego se hace mayor de cero en el momento 3 años. Esto se debe a que la generación de las nuevas crías sucede en el momento 3 años, lo cual a su vez se debe a que los especímenes comprados deben pasar por la etapa de Terneras tipo 1, luego Terneras tipo 2, luego por Terneras tipo 3, para luego pasar a generar nuevas crías, a su vez que pasan a ser Vacas tipo 1. Esta es la primera vez que se generan nuevos críos hembra por parte del ganado propio de la finca. Una tercera característica es que $N_{t n r 1}$ toma un comportamiento oscilatorio periódico, que va desde cero hasta 50. Esto se debe a la venta de crías hembra, teniendo en cuenta que si no se realizara venta de crías hembra se experimentaría un crecimiento indefinidamente creciente. 
Una cuarta característica es que el período de la oscilación es de 7 años. Es decir, si se toma un punto cualquiera de la evolución de $N_{t n r 1}$, en 7 años atrás o adelante se repite dicho punto.

Se puede ver que el valor de $N_{t n r 2}$ es $N_{t n r 1}$ retardado un año, lo cual se debe que los flujos para $N_{t n r 2}$ son los flujos de $N_{t n r 1}$ pero retardados. Esto es coherente con la realidad. Al igual que con el número de terneras tipo $1\left(N_{t n r 1}\right)$, también las variables $N_{t n r 2^{\prime}} N_{t n r 3^{\prime}}$ $N_{v c 1} \ldots N_{v c 5}$ sufren un comportamiento oscilatorio, que va desde cero hasta 50, con un período de 7 años.

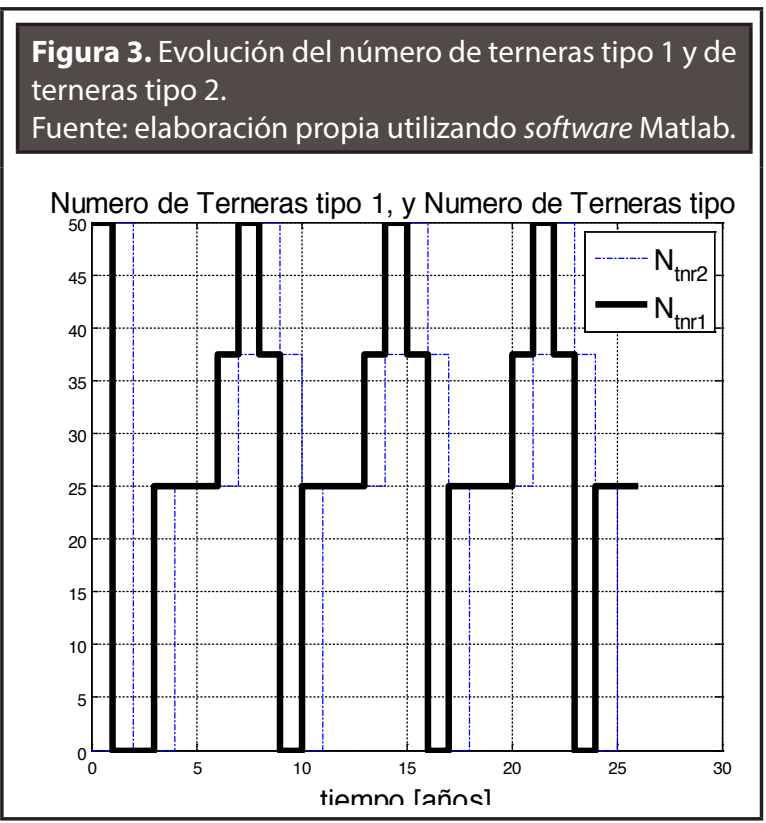

En la Figura 4 se muestra la evolución del número de Terneras tipo 1 hasta número de Vacas tipo 1 . Se puede ver que $N_{v c 1}, N_{t n r 3}, N_{t n r 2}$, tienen los mismos valores que $N_{t n r 1}$, pero retardados, con distintos retardos de tiempo. Por ejemplo, $N_{v c 1}$ es igual que $N_{t n r 1}$ pero retardado 3 años. Esto demuestra que los resultados son coherentes con la idea que se tuvo en el planteamiento de la evolución de las variables. Además es coherente con la realidad.

La Figura 5 muestra la evolución de número de Vacas tipo $1\left(N_{v c 1}\right)$, hasta número de Vacas tipo 5 $\left(N_{v c 5}\right)$. También en este caso se puede ver que las variables $N_{v c 1}$ hasta $N_{v c 5}$ tienen los mismos valores pero con retardo de tiempo.
Figura 4. Evolución del número de terneras tipo 1, hasta número de vacas tipo 1.

Fuente: elaboración propia, utilizando software Matlab.
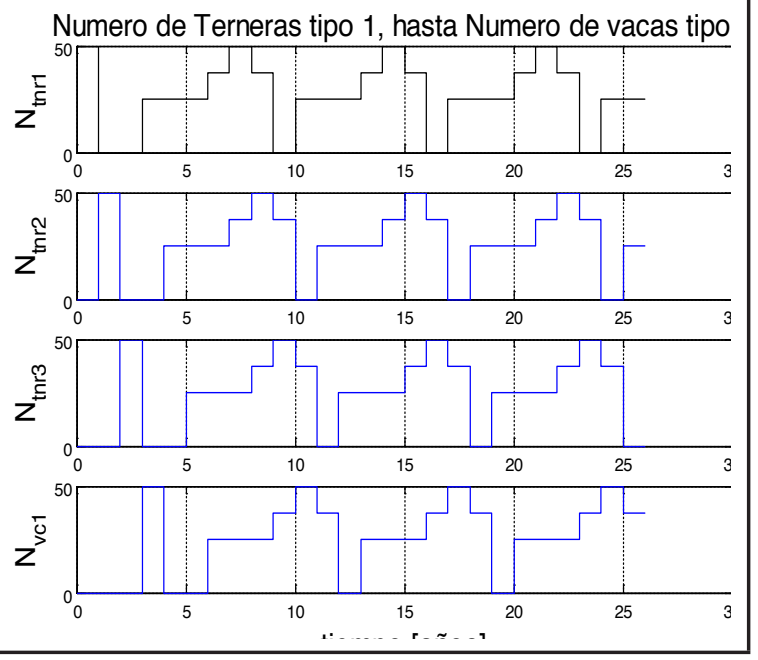

Figura 5. Evolución del número de vacas tipo $1\left(N_{v c c}\right)$, hasta número de vacas tipo $5\left(N_{v c 5}\right)$.

Fuente: elaboración propia utilizando software Matlab

Numero de Vacas tipo 1, hasta Numero de vacas tipo
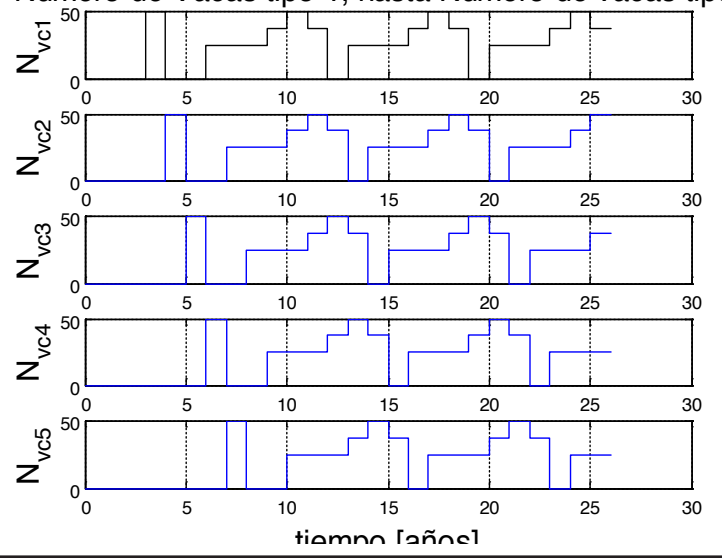

En la Figura 6 se muestra la evolución del número de ejemplares mayores de un año de edad $\left(N_{\text {eje1 }}\right), \mathrm{y}$ del número de Vacas productivas $\left(N_{v c}\right)$. Se puede ver que $N_{\text {ejer }}$, comienza en cero, y asciende lentamente hasta que en el instante $t=9$ años llega al valor límite $N_{\text {lim }}=200$ y se mantiene constante en dicho valor. Esto es coherente con el mecanismo de control utilizado, pues cuando hay un exceso de críos hembra, el mecanismo elimina los críos hembra de tal forma que $N_{\text {eje1 }}$ sea igual al número límite $N_{\text {lim }}$ y no lo sobrepase. 
El número de vacas productivas $\left(N_{v c}\right)$ comienza desde cero y asciende hasta que a los 9 años converge a un atractor periódico, es decir, a un comportamiento oscilatorio repetitivo. Una primera característica observada es que $N_{v c}$ nunca sobrepasa a $N_{\text {eje1 }}$, lo cual se debe a que $N_{e j e 1}$ comprende a $N_{v c}$ pero también a $N_{t n r 2}$ y $N_{t n r 3}$. Una segunda característica es que el valor de $N_{v c}$ se hace mayor que cero a partir de 3 años, lo cual se debe a que los especímenes comprados deben pasar por la etapa de Terneras 1, luego por Terneras 2 , Terneras 3, antes de pasar a Vacas tipo 1. Este recorrido implica una demora de tres años para que los especímenes comprados pasen a ser Vacas tipo 1. Estos aspectos son coherentes con la realidad.

Figura 6. Evolución del número de ejemplares mayores de un año $\left(N_{\text {ejel }}\right)$ y número de vacas productivas $\left(N_{v c}\right)$.

Fuente: elaboración propia utilizando software Matlab.

Numero de ejemplares mayores de un año $\left(\mathrm{N}_{\text {eje1 }}\right)$, y numero de vacas productivas $\left(\mathrm{N}_{\mathrm{vc}}\right)$

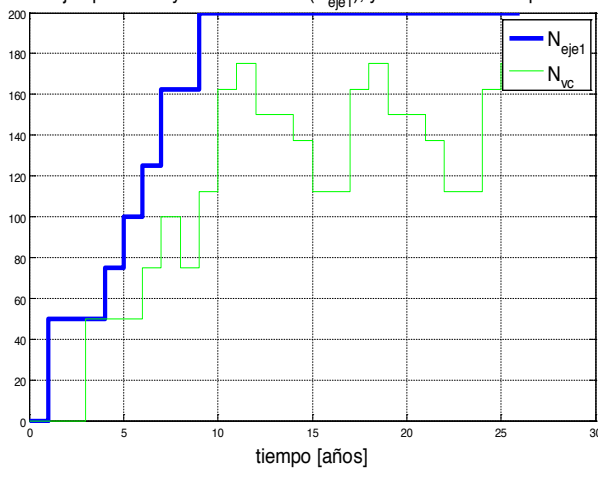

Figura 7. Evolución del número de críos hembra vendidas.

Fuente: elaboración propia utilizando software Matlab.

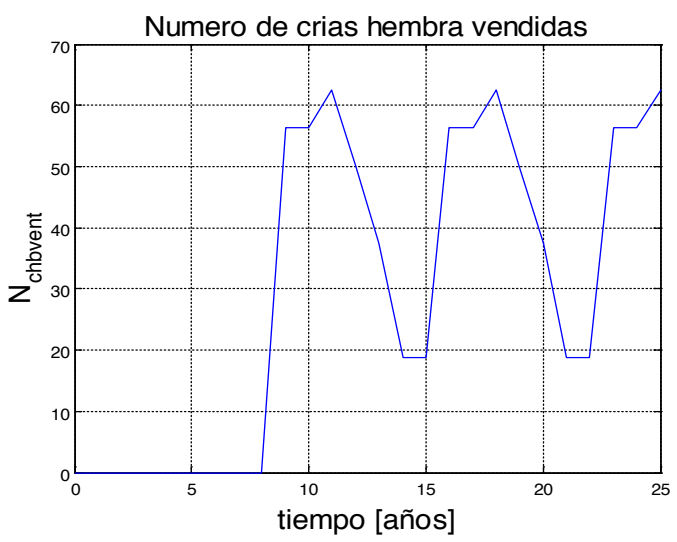

En la Figura 7 se puede ver la evolución del número de crías hembra vendidas. Su valor se hace mayor de cero a partir de 9 años y posteriormente se mantiene mayor que cero. Esto está relacionado con la alta producción de críos hembra, lo cual implica que se deben vender de manera continua.

\section{Comportamiento bajo condiciones extremas}

\section{de las entradas}

Para evaluar la calidad de las ecuaciones planteadas en tiempo discreto y la estructura causal, se pueden considerar situaciones extremas para las variables externas al modelo (v. Aracil, 1995: p. 64): para cada variable se toma un valor extremo bajo, un valor nominal y un valor extremo alto, ver Tabla 2.

TABLA 2. RESPUESTA ANTE VARIACIONES EN LOS PARÁMETROS INTERNOS DEL MODELO. SE USA MODELO EN TIEMPO DISCRETO, USANDO MATLAB. VALORES NOMINALES DE LAS VARIABLES: NÚMERO DE TERNERAS COMPRADAS EN TIEMPO INICIAL: $N_{\text {tnrto }}=50$; PORCENTAJES DE NACIMIENTOS DE HEMBRA EN LOS PARTOS DE LAS VACAS: $N_{\text {cmhb }}=50 \%$; MÁXIMO NÚMERO DE ESPECÍMENES MAYORES DE UN AÑO: $N_{\text {lim }}=200$.

\begin{tabular}{|c|c|c|c|}
\hline $\begin{array}{c}\text { Parámetro } \\
\text { sometido a } \\
\text { variación }\end{array}$ & $\begin{array}{c}\text { Valor del } \\
\text { parámetro } \\
\text { sometido a } \\
\text { variación }\end{array}$ & $\begin{array}{c}\text { Número } \\
\text { de vacas } \\
\text { productivas } \\
\left(N_{v c}\right)\end{array}$ & $\begin{array}{l}\text { Número de } \\
\text { Terneras } \\
\text { tipo } 1\left(N_{t n r 1}\right)\end{array}$ \\
\hline \multirow{3}{*}{$\begin{array}{c}\text { Terneras } \\
\text { compradas } \\
\text { en tiempo } \\
\text { inicial }\left(N_{\text {tnrto }}\right)\end{array}$} & 20 & 123 a 165 & 15 a 41 \\
\hline & 50 & 112 a 175 & 0 a 50 \\
\hline & 90 & 110 a 200 & 0 a 90 \\
\hline \multirow{3}{*}{$\begin{array}{c}\text { Porcentajes } \\
\text { de } \\
\text { nacimientos } \\
\text { de hembra } \\
\text { en los partos } \\
\text { de las vacas } \\
\left(N_{\text {ncmbb }}\right)\end{array}$} & $20 \%$ & 50 & 10 \\
\hline & $50 \%$ & 112 a 175 & 0 a 50 \\
\hline & $80 \%$ & 120 a 200 & 0 a 50 \\
\hline \multirow{3}{*}{$\begin{array}{c}\text { Máximo } \\
\text { número de } \\
\text { especímenes } \\
\text { mayores de } \\
\text { un año }\left(N_{l i m}\right)\end{array}$} & 90 & 40 a 90 & 0 a 45 \\
\hline & 200 & 112 a 175 & 0 a 50 \\
\hline & 300 & 175 a 250 & 25 a 68 \\
\hline
\end{tabular}

Lo que se concluye es que:

- Los tres parámetros sometidos a variación tienen un efecto significativo sobre el número 
de vacas productivas $\left(N_{v c}\right)$, y el número de terneras tipo $1\left(N_{t n r 1}\right)$.

- Un aumento en el número de terneras compradas en tiempo inicial $\left(N_{\text {tnrto }}\right)$, genera un aumento en el radio de oscilación de $N_{v c}$ y $N_{t n r 1}$. Esto implica que $N_{\text {tnrto }}$ no tiene un claro efecto de aumento neto o disminución neta de $N_{v c}$ y $N_{t n r 1}$.

- El porcentaje de nacimientos de hembra en los partos de las vacas $\left(N_{\text {ncmhb }}\right)$ tiene un efecto significativo sobre $N_{v c}$ y $N_{t n r 1}$. Un aumento en $N_{n c m h b}$ genera un aumento del límite inferior de la oscilación de $N_{v c}$ y $N_{t n r 1}$, lo cual implica que $N_{\text {ncmhb }}$ genera un aumento efectivo de $N_{v c}$ y $N_{t n r 1}$.

- Un aumento en $N_{\text {lim }}$ genera un aumento en el límite inferior de la oscilación de $N_{v c} \mathrm{y} N_{t n r 1}$, lo cual implica que el aumento de $N_{\text {lim }}$ efectivamente genera un aumento de $N_{v c}$ y $N_{t n r 1 .}$

\section{DISCUSIÓN Y CONCLUSIONES}

Los diagramas que se presentan en el texto de Schaffernicht (2006: pp. 217 y 218) fueron la base para plantear el diagrama de niveles y flujos y el correspondiente modelo matemático utilizando el software Matlab. El planteamiento del mapa de reservas y flujos involucró un alto número de re-planteamientos y mejoras, lo cual es una parte reconocida como crucial en la metodología de Dinámica de Sistemas.

Varias tareas se realizaron con base en el diagrama de niveles y flujos: i) el planteamiento de la explicación endógena; ii) la definición de variables endógenas y exógenas.

Al obtener la información suministrada por los dos expertos de la Universidad de Caldas, fue necesario reacomodar y arreglar los diagramas, principalmente las partes relacionadas con la forma de controlar que el número de ejemplares mayores de un año de edad no sobrepase el número límite.

Para plantear las ecuaciones en tiempo discreto no se utilizó ninguna ley de la física ni de la biología ni de la química. En su lugar, se utilizó la lógica de contar el número de ejemplares en cada una de las edades, cuando se tiene la siguiente situación: en un principio no se tiene ningún ejemplar en ninguna de las edades; luego se adquieren unos ejemplares que entran a la primera de las edades; luego estos ejemplares al cabo de un año, pasan a la segunda edad; luego al cabo de un año pasan a una tercera edad; y así sucesivamente. Esta lógica o esta situación fue el principio del planteamiento del modelo. Los niveles son el número de ejemplares en cada una de las edades, y los flujos son la cantidad de ejemplares que pasan entre un nivel y otro. Esta lógica o situación implica que: i) la cantidad de ejemplares en una edad es igual a la cantidad de ejemplares de la edad anterior, retardado un año, siempre y cuando no haya pérdidas o salidas; ii) cualquiera de los flujos, exceptuando el primer flujo, es igual al flujo que le precede, retardado un año. Por tanto, los flujos son retardados. El ejemplo presente en el texto de Schaffernicht (2006: pp. 217 y 218), fue la base para este planteamiento. El mencionado conteo se realiza con base en los flujos, pues se definen los flujos con base en el retardo de tiempo mencionado y luego con base en los flujos se definen los niveles. Luego se define la cantidad de nacimientos de terneros hembra y terneros macho y, por último, se plantea la acción de control de venta de terneras y venta de terneros, para evitar que el número máximo de ejemplares mayores de un año sobrepase el número límite.

Esta lógica fue planteada en forma de algoritmo, con el propósito de escribirlo luego en Matlab. Se utilizó un índice, al cual se le da el nombre de $k$. Los resultados de la simulación fueron coherentes con la realidad, de hecho no se obtuvieron valores negativos de las variables como número de terneras tipo 1 , número de terneras tipo 2 , número de vacas y número de ejemplares mayores de un año de edad, entre otras.

En todo el trabajo del presente estudio se puede observar que se aplican conceptos propios del Diseño de sistemas de control automático, por ejemplo la definición de la condición de balance o refuerzo, para el lazo de realimentación. Además, se puede observar que el proceso es iterativo circular, como lo señala Sterman. De hecho, las modificaciones en el mapa de reservas y flujos implicaron la realiza- 
ción de modificaciones en el resto de tareas, principalmente en la explicación endógena, en la tabla de frontera de modelo y en el modelo matemático.

La figura de la evolución de número de número de Terneras tipo $1\left(N_{t n r 1}\right)$ y número de Terneras tipo $2\left(N_{t n r 2}\right)$, nos permite concluir lo siguiente:

- $\quad$ El valor de $N_{t n r 1}$ comienza en 50, esto se debe a que este es el número de especímenes comprados en el tiempo inicial.

- $\quad N_{t n r 1}$ se hace cero y luego se hace mayor de cero en el momento 3 años. Esto se debe a que los especímenes comprados deben pasar por la etapa de Terneras tipo 1, luego Terneras tipo 2 , luego por Terneras tipo 3, para luego pasar a reproducir críos, a su vez que pasan a ser Vacas tipo 1. Esta es la primera vez que se generan críos hembra por parte del ganado propio de la finca. La generación de las crías sucede en el momento 3 años.

- $\quad N_{t n r 1}$ toma un comportamiento oscilatorio periódico, que va desde cero hasta 50. Esto se debe a la venta de crías hembra, pues de lo contrario experimentaría un crecimiento indefinidamente creciente.

- $\quad$ El período de la oscilación es de 7 años. Es decir, si se toma un punto cualquiera de la evolución de $N_{t n r 1}$, en 7 años atrás o adelante se repite dicho punto.

- $\quad$ El valor de $N_{t n r 2}$ es igual a $N_{t n r 1}$ retardado un año, lo cual se debe que los flujos para $N_{t n r 2}$ son los flujos de $N_{t n r 1}$ pero retardados.

La figura de la evolución del número de ejemplares mayores de un año de edad $\left(N_{e j e 1}\right)$, y del número de Vacas productivas $\left(N_{v c}\right)$, permite concluir que:

- $\quad$ El número de vacas productivas $\left(N_{v c}\right)$ nunca sobrepasa a $N_{e j e 1}$, lo cual se debe a que $N_{e j e 1}$ comprende a $N_{v c}$ pero también a $N_{t n r 2}$ y $N_{t n r 3}$.

- $\quad$ El valor de $N_{v c}$ se hace mayor que cero a partir de 3 años, lo cual se debe a que los especímenes comprados deben pasar por la etapa de Terneros 1, luego por Terneros 2, luego por
Terneros 3, antes de pasar a Vacas tipo 1. Este recorrido implica una demora de tres años para que los especímenes comprados pasen a ser Vacas 1.

- $\quad$ El número de ejemplares mayores de un año $\left(N_{e j e 1}\right)$, comienza en cero, asciende, hasta que en 9 años llega hasta el límite $\left(N_{\text {lim }}\right)$ y se mantiene en dicho valor. Esto es coherente con el mecanismo de control utilizado, pues cuando hay un exceso de críos hembra, el mecanismo elimina los críos hembra de tal forma que $N_{\text {eje1 }}$ sea igual al número límite $N_{\text {lim }}$ y no lo sobrepase.

La tabla de respuesta ante variaciones en los parámetros internos del modelo, Tabla 2 , se obtuvo con base en simulaciones. Se concluye que:

- El número de terneras compradas en tiempo inicial $\left(N_{\text {trrto }}\right)$ tiene un efecto significativo sobre el número de vacas productivas $\left(N_{v c}\right)$, número de terneras tipo $1\left(N_{t n r 1}\right)$ e ingresos por leche $\left(I_{\text {leche }}\right)$. Un aumento en $N_{\text {tnrto }}$ genera un aumento en el radio de oscilación de $N_{v c}$ y $N_{t n r r}$, de tal modo que el límite inferior de la oscilación disminuye y el límite superior aumenta. De esta forma, no hay un efecto de aumento neto o disminución neta de $N_{v c} \mathrm{y} N_{t n r}$.

- $\quad$ El porcentaje de nacimientos de hembra en los partos de las vacas $\left(N_{n c m h b}\right)$, tiene un efecto significativo sobre $N_{v c^{\prime}} N_{t n r 1}$ e $I_{\text {leche }}$. Un aumento en $N_{\text {ncmhb }}$ genera un aumento del límite inferior de la oscilación de $N_{v c}$ y $N_{t n r 1}$ lo cual implica que hay un aumento efectivo de $N_{v c}$ y $N_{t n r}$.

El máximo número de especímenes mayores de un año, $N_{\text {lim }}$, también tiene un efecto significativo sobre $N_{v c}$ y $N_{t n r 1}$. Un aumento en $N_{\text {lim }}$ genera un aumento en el límite inferior de la oscilación de $N_{v c}$ y $N_{t n r 1}$, lo cual implica que el aumento de $N_{\text {lim }}$ efectivamente genera un aumento de $N_{v c}$ y $N_{t n r 1}$.

En la Tabla 2, la respuesta ante variaciones en el número de terneras compradas en tiempo inicial fue obtenida con base en simulaciones. Se puede concluir lo siguiente: 
- $\quad$ Para valores de $N_{\text {tnrto }}$ entre 1 a 90, el aumento de $N_{\text {tnrto }}$ genera una disminución en el tiempo de estabilización para $N_{t n r 1}, N_{v c^{\prime}}$ y $N_{\text {eje1 }}$.

- $\quad$ Para $N_{\text {tnrto }}=50$ y $N_{\text {tnrto }}=90$, el tiempo de estabilización para $N_{t n r 1}$ es cero, lo cual significa que $N_{t n r 1}$ no converge hacia el comportamiento oscilatorio, sino que comienza en este.

Algunas diferencias del presente trabajo con respecto al trabajo de Chajin y Jiménez (2010):

- El modelamiento matemático se planteó a tiempo discreto, de modo que para la simulación se empleó un ciclo for y el índice $k$.

- Se utilizó el método de cadena con flujos retardados, para la definición de las variables tipo flujo y las variables tipo nivel.

- El control del número de ejemplares mayores de un año de edad se realizó con base en venta de críos hembra y el cálculo del índice de prueba $N_{p b f t} l_{k^{\prime}}$ el cual se define como el valor, que para un momento $k$, toma el número de ejemplares mayores de un año de edad que existirían en un momento futuro $k+1$, bajo la condición de no venta de críos hembra.

El presente trabajo tiene las siguientes contribuciones:

- Desarrollo del mapa de niveles y flujos para el sistema ganadero de Laguna Negra.

- Desarrollo del modelo matemático a tiempo discreto, para el caso específico del sistema ganadero de Laguna Negra, utilizando el método de cadena con flujos retardados, para la definición de las variables tipo flujo y tipo nivel.

- $\quad$ Planteamiento del control del número de ejemplares mayores de un año de edad, basado en venta de críos hembra y el cálculo del índice de prueba $\left.N_{p b f t}\right|_{k^{\prime}}$ el cual se define como el valor, que para un momento $k$, toma el número de ejemplares mayores de un año de edad que existirían en un momento futuro $k+1$, bajo la condición de no venta de críos hembra.
- Análisis del efecto de variables exógenas sobre el comportamiento de las variables de nivel, mediante simulación, para el caso específico del sistema ganadero de Laguna Negra.

La posibilidad de trabajo futuro es tener en cuenta variables biológicas como clima y otras del comportamiento del ganado; esto le daría una alta rigurosidad al estudio y correspondencia con la realidad. Esto implicaría reescribir y rehacer todo el estudio, incluyendo las siguientes tareas:

- Revisión de literatura para analizar e identificar nuevas variables biológicas.

- $\quad$ Re-definición de las nuevas variables biológicas.

- Determinación de los valores de las nuevas variables biológicas, para el caso de Laguna Negra.

- Re-definición de los datos para el diagrama de niveles y flujos.

- Re-definición de las tablas de frontera del modelo.

- $\quad$ Realización del nuevo diagrama de niveles y flujos.

- Re-formulación de todo el modelo matemático, con inclusión de las nuevas variables.

- Modificación de las simulaciones.

A este fin, sería importante analizar a profundidad el trabajo de el trabajo de Chajin y Jiménez (2010) y utilizar las variables biológicas allí utilizadas.

\section{REFERENCIAS}

Aracil, J. (1999). On the Qualitative Properties in System Dynamics Models. European Journal of Economic and Social Systems, 13(1), p.p. 1-18.

(1995) Dinámica de sistemas. Madrid, España: Isdefe. $87 \mathrm{p}$.

Bozic, M. (2009). An Econometric Analysis of U.S. Milk Production: a Herd Dynamics Model. [pdf] Italia: Universidad de Trento. Disponible en: http://marinbozic. info/PDF/Bozic_MAThesis.pdf, [Consultado el 13 de octubre de 2015].

Carvajal, S. y Arango, S. (2011). Simulación para la evaluación de una propuesta de remuneración del servicio de arranque autónomo en Colombia. Revista Facul- 
tad de Ingeniería Universidad de Antioquia, 1(59), p.p. 267-276.

Carvajal, S., et al. (2011). A Study of Incentives to Increase the Use of DG on Colombia Based on a System Dynamics Modeling. Ingeniería e Investigación, 31(2), p.p. 58-65.

Chajin, V. y Jiménez, N. (2010). Modelo de simulación de ganadería bovina intensiva: SIPROBI 1.0. [pdf] Colombia: Universidad Industrial de Santander. Disponible en: http://repositorio.uis.edu.co/jspui/ bitstream/123456789/2691/2/134018.pdf [Consultado el 13 de octubre de 2015].

Dieguez-Cameroni, F. y Terra, R. (2014). Aplicación del modelo de una explotación ganadera extensiva (MEGanE) para el estudio de la sensibilidad de la producción ganadera a la amplitud de la variabilidad de la oferta de forraje. [en línea] Buenos Aires, Argentina: Sociedad Argentina de Informática e Investigación Operativa. Disponible en: http://sedici.unlp.edu.ar/ handle/10915/41980.

Fundación Pangea y Corpocaldas. (2007) Informe de la caracterización de humedales en el proyecto Conservación de Humedales Altoandinos en cinco municipios del departamento de Caldas: Neira, Salamina, Villamaría, Manizales y Marulanda. Manizales, Colombia: Fundación Pangea y Corpocaldas.

García, J. M. (2003) Teoría de ejercicios prácticos de dinámica de sistemas. Barcelona, España. 292 p.

Murgueitio, E. (1999). Reconversión ambiental y social de la ganadería bovina en Colombia. World Animal Review, 93(2), p.p. 2-15.

Schaffernicht, M. (2006). Indagación de situaciones complejas mediante la dinámica de sistemas. Talca, Chile: Universidad de Talca. $254 \mathrm{p}$.

Sterman, J. (2000) Business Dynamics: Systems Thinking and Modeling for a Complex World. New York, USA: McGraw Hill.

Tedeschi, L.; Nicholson, C. y Rich, E. (2011). Using System Dynamics modeling approach to develop management tools for animal production with emphasis on small ruminants. Small Ruminant Research, 98(1-3), June, p.p. 102-110.

Toro, D., et al. (2012). Estudio limnológico de la Laguna Negra: zona amortiguadora del PNN Los Nevados. Boletín Científico Centro de Museos Museo de Historia Natural, 16(2), p.p. 23-38.

\section{PARA CITAR ESTE ARTÍCULO TO REFERENCE THIS ARTICLE / PARA CITAR ESTE ARTIGO /}

Rincón, A,; Florez, G.Y.; Redondo, Y.M.; Olivar, G. (2015). Modelo matemático de la demografía del ganado de un predio del sector El Ocho Letras. Revista EIA, 12(24), julio-diciembre, pp. 101-119. [Online]. Disponible en: DOI: http:/dx.doi. org/10.14508/reia.2015.12.24.101-119 